\title{
BMJ Open Cluster randomised controlled trial of a financial incentive for mothers to improve breast feeding in areas with low breastfeeding rates: the NOSH study protocol
}

Clare Relton, ${ }^{1}$ Mark Strong, ${ }^{2}$ Mary J Renfrew, ${ }^{3}$ Kate Thomas, ${ }^{2}$ Julia Burrows, ${ }^{2}$ Barbara Whelan, ${ }^{1}$ Heather M Whitford, ${ }^{3}$ Elaine Scott, ${ }^{1}$ Julia Fox-Rushby, ${ }^{4}$ Nana Anoyke, ${ }^{4}$ Sabina Sanghera, ${ }^{4}$ Maxine Johnson, ${ }^{1}$ Easton Sue, ${ }^{1}$ Stephen Walters ${ }^{2}$

To cite: Relton C, Strong M, Renfrew MJ, et al. Cluster randomised controlled trial of a financial incentive for mothers to improve breast feeding in areas with low breastfeeding rates: the NOSH study protocol. BMJ Open 2016;6:e010158. doi:10.1136/bmjopen-2015010158

- Prepublication history for this paper is available online. To view these files please visit the journal online (http://dx.doi.org/10.1136/ bmjopen-2015-010158).

Received 1 October 2015 Revised 24 November 2015 Accepted 2 December 2015

CrossMark

For numbered affiliations see end of article.

Correspondence to

Dr Clare Relton;

c.relton@sheffield.ac.uk

\section{ABSTRACT}

Introduction: Breast feeding can promote positive long-term and short-term health outcomes in infant and mother. The UK has one of the lowest breastfeeding rates (duration and exclusivity) in the world, resulting in preventable morbidities and associated healthcare costs. Breastfeeding rates are also socially patterned, thereby potentially contributing to health inequalities. Financial incentives have been shown to have a positive effect on health behaviours in previously published studies.

Methods and analysis: Based on data from earlier development and feasibility stages, a cluster (electoral ward) randomised trial with mixed-method process and content evaluation was designed. The 'Nourishing Start for Health' (NOSH) intervention comprises a financial incentive programme of up to 6 months duration, delivered by front-line healthcare professionals, in addition to existing breastfeeding support. The intervention aims to increase the prevalence and duration of breast feeding in wards with low breastfeeding rates. The comparator is usual care (no offer of NOSH intervention). Routine data on breastfeeding rates at $6-$ 8 weeks will be collected for 92 clusters (electoral wards) on an estimated 10833 births. This sample is calculated to provide $80 \%$ power in determining a $4 \%$ point difference in breastfeeding rates between groups. Content and process evaluation will include interviews with mothers, healthcare providers, funders and commissioners of infant feeding services. The economic analyses, using a healthcare provider's perspective, will be twofold, including a within-trial cost-effectiveness analysis and beyond-trial modelling of longer term expectations for cost-effectiveness. Results of economic analyses will be expressed as cost per percentage point change in cluster level in breastfeeding rates between trial arms. In addition, we will present difference in resource use impacts for a range of acute conditions in babies aged 0-6 months.

Ethics and dissemination: Participating organisations Research and Governance departments approved the study. Results will be published in peer-reviewed journals and at conference presentations.

Trial registration number: ISRCTN44898617; Preresults.

\section{INTRODUCTION}

\section{Breast feeding}

The $\mathrm{WHO}^{1}$ recommends that babies are exclusively breast fed until 6 months, with breast feeding (BF) continuing for up to 2 years after solid foods have been introduced. This recommendation is supported by all four UK Departments of Health and is based on evidence regarding the long-term and shortterm benefits of $\mathrm{BF}^{2}{ }^{3}$ Despite this policy position, and an increase in numbers of women starting to breast feed, ${ }^{4} 6$-8-week BF rates in the UK have remained low for several decades in contrast to some other developed countries (eg, Norway, Sweden) where the majority of women breast feed for at least 2 months and many for longer. Because infant feeding is socially patterned with women from lowincome groups having the lowest rates, low $\mathrm{BF}$ rates also have a serious negative impact on inequalities in health.

\section{Financial incentives for behaviour change}

Financial incentives have been shown to be effective in promoting a range of positive health behaviours ${ }^{5}$ including adopting a healthy diet. ${ }^{6}$ Women on unemployment benefit in the Quebec province of Canada have routinely been offered financial incentives ( $\$ 55$ per month) for $\mathrm{BF}$ since the mid-1990s, ${ }^{7}$ but there has been no formal 
evaluation of this or any other financial incentive scheme for BF.

If financial incentives were found to be acceptable, effective in increasing $\mathrm{BF}$ rates and cost-effective, this would have implications for future health policy. For example, Pokhrel et $a l^{8}$ and Renfrew et $a l^{9}$ estimate that over $£ 17$ million could be saved each year in the UK, through reduced hospital admissions and fewer visits to general practitioners (GPs) relating to four acute child health conditions if exclusive $\mathrm{BF}$ rates were to increase from $7 \%$ to $45 \%$ at 4 months and if babies fed breast milk at discharge from neonatal units increased from $35 \%$ to $75 \%$. The conditions examined include gastrointestinal (GI) infection, acute otitis media, lower respiratory tract infection and necrotising enterocolitis. In addition, the cost-savings and value of life-time health gains for mothers from associated reductions in breast cancer were estimated to exceed £31million for each annual cohort of women. ${ }^{9}$ Childhood obesity, sudden infant death and cognitive outcomes-all conditions with important economic implications-were also found to be adversely affected by not BF, though given the nature of the available evidence it was not possible to attribute specific cost-savings from increased BF rates with regard to these conditions. ${ }^{9}$

\section{BF and public health}

Increasing $\mathrm{BF}$ rates is a priority in all four UK countries. In England, BF is a priority White Paper public health policy with the potential to impact on health inequalities, and the 6-8-week BF rate is an outcome in the Public Health Outcomes Framework. ${ }^{10} \mathrm{BF}$ is also a Department of Health 'Vital Signs' target ${ }^{10}$ and one of 20 key National Health Service (NHS) operating plan performance measures. The National Institute for Health and Care Excellence (NICE) guidance ${ }^{11}$ recommends a multistrategic approach to increase $\mathrm{BF}$ rates. There are therefore strong drivers within the NHS for the identification of successful strategies to improve $\mathrm{BF}$ rates.

\section{Results of development and feasibility testing stages}

This trial forms the third stage of a research project exploring the potential of financial incentives to increase $\mathrm{BF}$ in areas with low rates.

The first stage developed the idea, assessed the acceptability of the financial incentive 'shopping vouchers for BF' scheme in principle, agreed the components of the scheme with local women ${ }^{12}$ and midwives, health visitors,${ }^{13}$ and obtained permissions from the relevant authorities to test the scheme.

The second stage assessed the feasibility (acceptability and implementation) of the scheme in the real world in three small areas (an electoral ward in North Derbyshire, a 'neighbourhood' in Sheffield and a township in Rotherham). The main findings ${ }^{14}$ were that midwives and health visitors were willing to alert women to the scheme, and co-sign application forms and voucher claims; and that women joined the Nourishing Start for
Health (NOSH) scheme, claimed vouchers and preferred supermarket and high street vouchers to vouchers for local independent shops.

\section{Aim of the study}

The aim of the study is to evaluate the effectiveness of a financial incentive scheme (NOSH) designed to increase $\mathrm{BF}$ rates in wards with low $\mathrm{BF}$ rates using a cluster (electoral ward) randomised trial with mixed-method process and content evaluation.

\section{METHODS, DESIGN AND ANALYSIS Study objectives}

The primary objective is to test the impact of the offer of the NOSH intervention on $6-8$ weeks BF rates in clusters (with low $\mathrm{BF}$ rates $<40 \% \mathrm{BF}$ at $6-8$ weeks).

The secondary objectives are to: (1) test the impact of the intervention on BF rates at initiation in clusters with low BF rates; (2) examine the impact of the intervention on a range of disease outcomes, and impact on health service use in children aged 0-6 months; (3) determine the resource use and costs of providing the intervention by BF initiation, $6-8$ weeks and 6 months; (d) examine the cost-effectiveness of the intervention (incremental cost per percentage point change in $\mathrm{BF}$ ) at $6-8$ weeks in wards with low $6-8$-week BF rates $(<40 \%$ at $6-8$ weeks), accounting for costs associated with a monetary offer made up to 6 months postbirth and costs saved in children aged 0-6 months; and (e) examine the interaction between the effectiveness and cost-effectiveness of the intervention and a range of ward-level characteristics, to estimate the incremental cost-effectiveness of intervention over an extended time horizon, using an economic model-based analysis.

\section{Study design and setting}

The trial design is an open pragmatic cluster randomised controlled trial (cRCT) with a mixed-methods process and context evaluation and an economic evaluation. Clusters (2011 electoral wards) are randomised to either: (1) offer of a financial incentive scheme to women who live in a designated cluster (intervention arm) or (2) no offer (control arm; figure 1).

Districts are defined as the whole (or sometimes part) of a local government council (eg, Metropolitan Borough Council, City Council or a County Council). In order for a district to be eligible, the district must have:

- Electoral wards with low BF rates $(<40 \%$ at $6-8$ weeks);

- Not be currently providing financial incentives to breast feed;

- Provide approvals for midwives and health visitors to help deliver the scheme.

\section{The intervention}

The intervention to be trialled is a behaviour change intervention in the form of the offer of a structured financial incentive (shopping vouchers each worth 


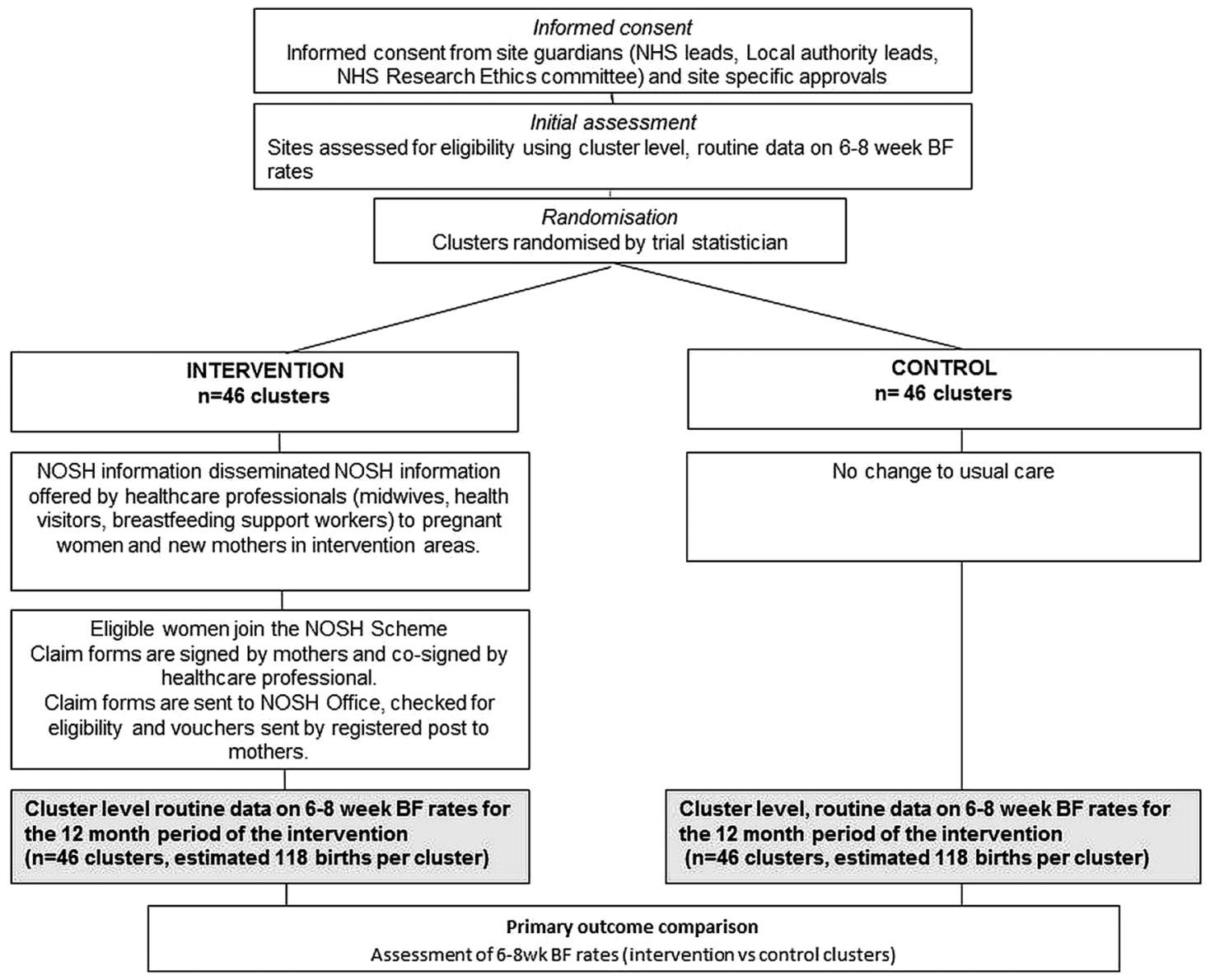

Figure $1 \mathrm{NOSH}$ trial schema. BF, breastfeeding; NOSH, Nourishing Start for Health; NHS, National Health Service.

$£ 40 \times 5$ ) to women over a 6 -month period-the NOSH scheme. The intervention will be introduced into the clusters randomised to the offer group through the distribution of NOSH scheme posters and booklets (via children's centres, GP surgeries, post offices and other public places in the intervention cluster wards); a press release to the local media, briefing notes and invitations to attend induction sessions about the NOSH scheme to all healthcare providers involved in the provision of infant feeding support services in the intervention clusters. These include midwives, health visitors, $\mathrm{BF}$ support workers and BF peer support workers. Information and materials about the NOSH scheme are provided to midwifery and health visiting teams working in the trial intervention wards. Midwives and health visitors will discuss the NOSH scheme with women during routine contact. Taking part in the NOSH scheme is voluntary; women are able to freely join and leave the NOSH scheme. To join the NOSH scheme women complete an application form which must be co-signed by their healthcare provider. On approving the application form, the NOSH office (based at the University of Sheffield) will forward a 'welcome pack'. This contains five NOSH claim forms for vouchers each worth $£ 40$ to be signed when the baby is 2 days, 10 days, 6 weeks, 3 months and 6 months) if the baby is still $\mathrm{BF}$ and/or receiving breast milk. The 'welcome pack' also contains a NOSH fridge magnet, and the NOSH booklet (figure 2) detailing information about the scheme and details of local support services in case of problems. Women will sign and date each NOSH claim form if her baby is still receiving breast milk and ask their healthcare provider to co-sign the NOSH claim form. The NOSH office will aim to send vouchers to mothers by return post. The intervention will be offered for babies born between 17 February 2015 and 17 February 2016.

\section{Verification of BF in order to receive vouchers}

The current method used in the UK to collect routine data on infant feeding relies on information exchanged between mother and her healthcare provider. The consensus from the extensive consultation with local stakeholders during an earlier stage of the NOSH project was that verification of BF should be confirmed by a signed statement from the mother and a signed statement from their healthcare provider on the voucher claim forms.

If a healthcare provider has concerns that the baby is not receiving any breast milk, then the healthcare provider can complete and send in a separate 'expression of concern form' detailing their concerns. However, it is vital that the NOSH scheme does not compromise the existing relationship between the mother and the 
healthcare provider, thus all claims will be paid regardless of whether or not concerns have been expressed by the healthcare provider. Analysis of the 'expression of concern' cards will inform understanding of the reliability of the verification method.

\section{Sampling frame and recruitment}

The sampling frame for the trial is clusters (electoral wards) with low $\mathrm{BF}$ rates $(<40 \%$ at $6-8$ weeks) in five districts (Sheffield, North Derbyshire, Rotherham, Doncaster and Bassetlaw). Clusters (2011 electoral wards) in each district were screened for eligibility using the most recent data on $\mathrm{BF}$ available, when planning the trial. Following randomisation, a total of 92 clusters (figure 3) were included in the trial with a total estimated number of 10833 births for the 1-year trial period.

\section{Inclusion and exclusion criteria for scheme participants}

All women aged 16 years and over, ordinarily resident in each ward, and with an estimated date of delivery between 18 February 2015 and before the end of the trial period on 17 February 2016 will be eligible to apply to join the NOSH scheme. No exclusion criteria are stipulated; exclusion will be determined on a case-by-case basis by healthcare providers using their clinical judgement.

\section{Randomisation}

The cluster random allocation sequence was generated by the study statistician (SW), who was not involved in the enrolment of clusters, using computer-generated random numbers, stratified by district, of variable block size. The random allocation sequence was implemented by CR who assigned the clusters (wards) to the

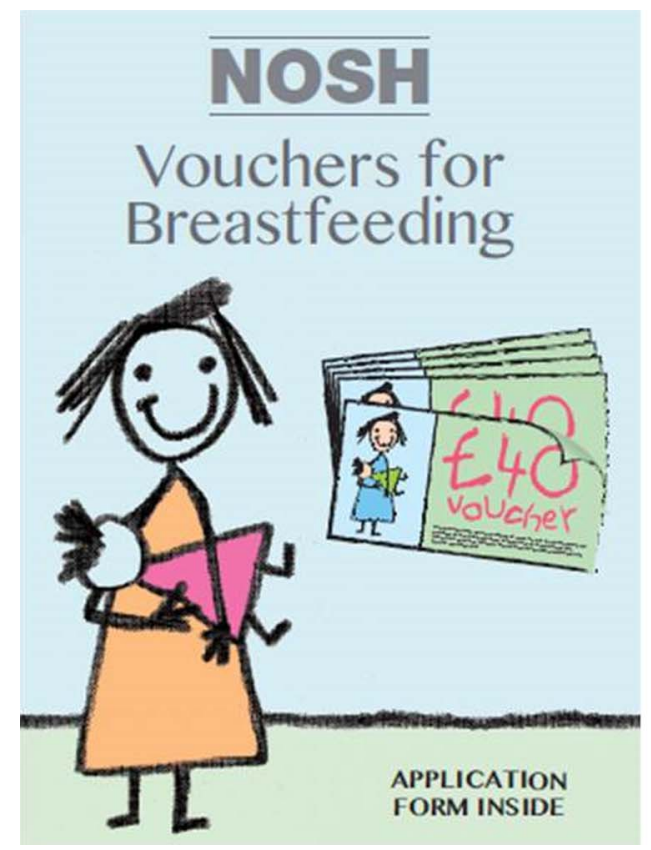

interventions. There is no blinding of trial participants, care providers, outcome assessors or data analysts.

\section{Outcomes}

The primary outcome measure is the cluster-level 6-8-week BF period prevalence over the intervention time period (1 April 2015 to 31 March 2016). BF will be defined as any BF (and will include babies who are receiving supplementary food as well as BF, and exclusively breastfed babies). The primary outcome will use routine 6-8 week BF data analysed at cluster (electoral ward) level. Secondary outcomes include BF initiation period prevalence; exclusive $\mathrm{BF}$ rate; number and length of admissions to hospital with: GI tract infection, otitis media, respiratory tract infection, necrotising enterocolitis and any (all) hospital admissions.

\section{Data collection}

Data used in this study will come from several sources, measured at a cluster level (defined by postcode) including local routine data from district public health departments and local NHS Trusts on BF rates, census data and Hospital Episode Statistics (HES) with further linkage to health resource groups. We will use routine data to determine cluster-level 6-8-week BF prevalence (these data are based on healthcare provider's professional judgement, after discussion with the mother). Cluster (ward)-level descriptive data will be collected using demographic data from the 2011 census, midyear population estimates and deprivation data from the English Index of Deprivation. Cluster-level covariates will include deprivation (Index of Multiple Deprivation (IMD) 2010), mother's age, ethnicity, birth rate (from local routine data sources), 6-8-week BF rate routine

\section{What do I get?}

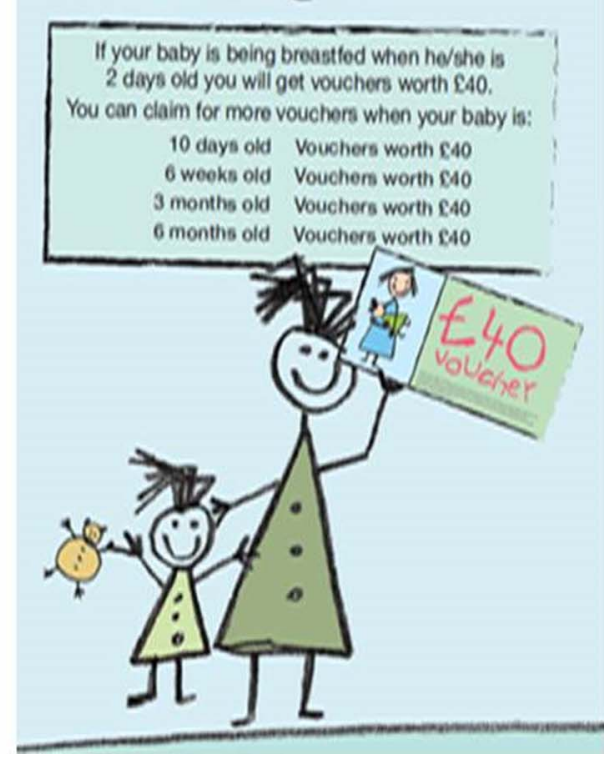

Figure 2 NOSH vouchers for breastfeeding booklet. NOSH, Nourishing Start for Health. 


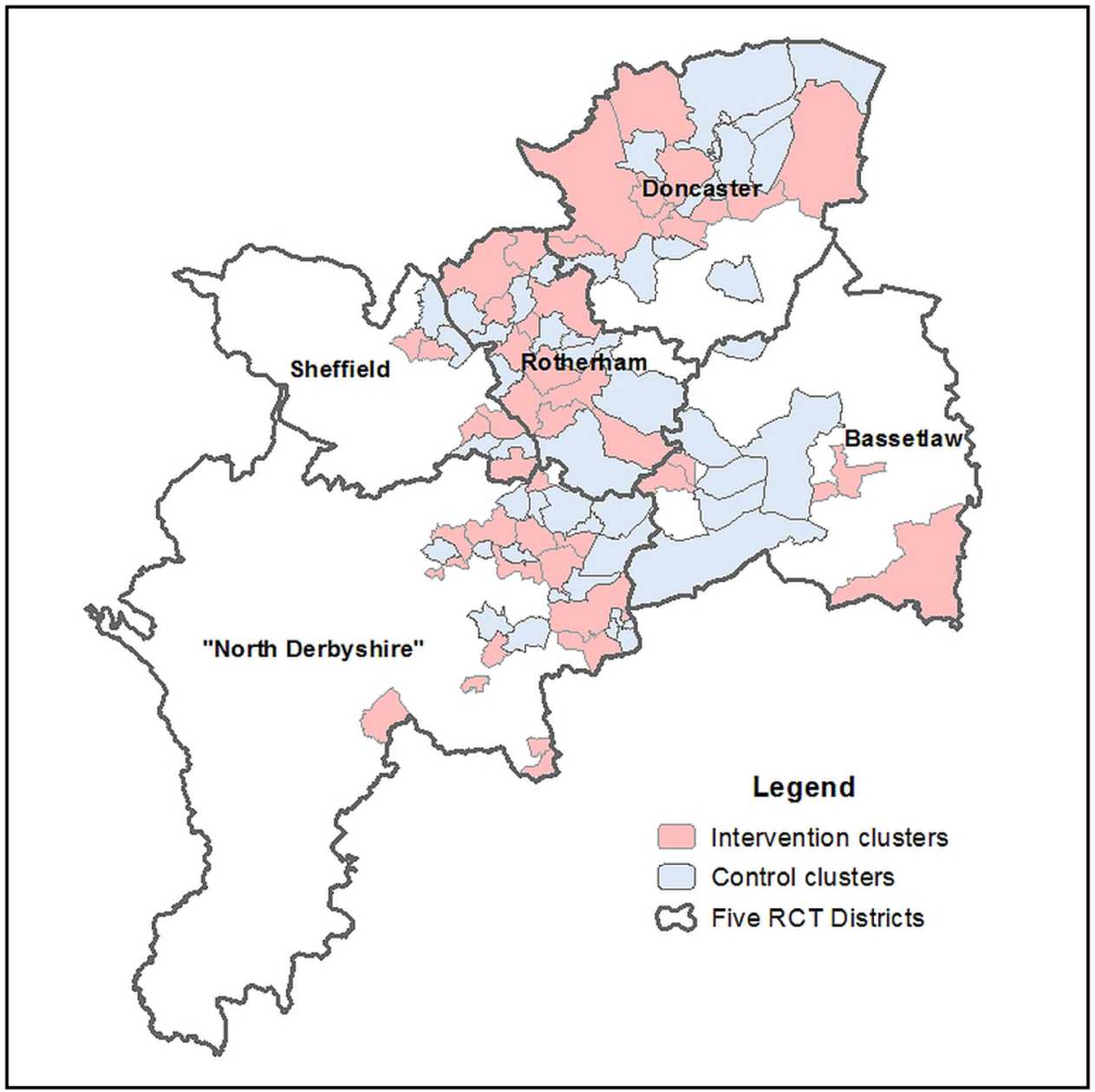

Figure 3 RCT districts, intervention and control clusters (electoral wards). RCT, randomised controlled trial.

data collected from public health and child health information services. Cluster secondary outcome measures from routine data will include $\mathrm{BF}$ initiation and exclusive $\mathrm{BF}$, and number and length of hospital admissions.

\section{Risk to accessing primary outcome data}

The transfer of the commissioning of the 0-5 Healthy Child Programme from the NHS to local authorities in October 2015 may impact on the trial's access to routine 6 -8-week BF data. Although national systems are being set up to ensure good routine data collection for 68-week $\mathrm{BF}$ prevalence, local organisational challenges may mean that data availability and quality varies by district/provider.

\section{Sample size}

The primary outcome measure is cluster-level 6 -8-week $\mathrm{BF}$ period prevalence over the intervention time period. The intra class correlation (ICC) $(0.01)$ was estimated from the most recently available 6 - 8 -week $\mathrm{BF}$ rates in the clusters in the sampling frame. Based on data from 92 clusters and an estimated 10833 births per year, the mean cluster size was 118 . The proportion of babies being breast fed at $6-8$ weeks was estimated as $27.6 \%$ (2985/10 833).
Assuming a $4 \%$ point increase in 6-8-week BF rates between the intervention and comparator groups represents a clinically/practically important difference; an ICC of 0.01 ; average cluster size of 118 births and a mean $6-8$-week BF rate of $28 \%$ in the control arm, then with 4463 births per group (8926 in total) the trial is powered to detect a $4 \%$ point increase in $\mathrm{BF}$ rates (from $28 \%$ to $32 \%$ ) as statistically significant at the 5\% (twosided) level and $80 \%$ power. For a cRCT, this will require a minimum of 76 clusters to be randomised (38 clusters per group).

\section{Data analysis plan \\ Statistical methods}

As the trial is a parallel group cRCT, with a usual (control) treatment arm, data will be reported and presented according to the revised CONSORT statement for cRCTs. ${ }^{15}$ All statistical exploratory tests will be twotailed with $\alpha=0.05$. The analysis will be performed on an intention to treat basis. The analysis of the outcome data will be carried out at the cluster level, using aggregate cluster-level summary data on $\mathrm{BF}$ rates for each cluster, as we will not have individual-specific mother-level outcome data. 
The primary objective is to evaluate the clinical effectiveness of the intervention (NOSH Scheme) compared with a usual care control group, in new mothers, on BF rates at $6-8$ weeks in clusters with low $\mathrm{BF}$ rates $(<40 \%$ at 6-8 weeks). Aggregate cluster-level summary BF rates, at $6-8$ weeks will be compared, between the intervention and control groups, using a multiple linear regression model with regression coefficients estimated by weighted ordinary least squares.

The primary analysis will be a multiple linear regression model with terms for the baseline cluster $\mathrm{BF}$ rate, district, randomised group and will be weighted with a weight which is proportional to the inverse of the variance of the estimated $\mathrm{BF}$ rate outcome. The effectiveness of the NOSH intervention in the intervention period will be tested by the size and significance of the group term in the multiple linear regression model. A $95 \%$ CI for the group term for BF at 6-8 weeks between the intervention and control group will be reported, from the model, along with its associated $p$ value.

A sensitivity analysis will be performed alongside this primary analysis and will include additional baseline cluster-level covariates, such as cluster-level deprivation (IMD 2010); cluster-level age and ethnicity as defined above; cluster-level birth rate; cluster-level maternal smoking rate at delivery in the multiple linear regression model. Again a $95 \%$ CI for the group term for BF at 6-8 weeks between the intervention and control group will be reported, from the model, along with its associated $\mathrm{p}$ value. This estimate will be plotted alongside the primary analysis estimate in a meta-analysis-style forest plot graph. Secondary cluster-level outcomes (eg, BF initiation) will be analysed in a similar way with a similar model for the primary outcome. Any missing cluster-level primary outcome (BF at 6-8 weeks) data will be imputed using a variety of imputation methods including last observation carried forward, regression and multiple imputation.

An exploratory subgroup analysis will be performed using multiple linear regression with the primary outcome, summary $\mathrm{BF}$ rates at $6-8$ weeks, as the response. We will use an interaction statistical test between the randomised intervention group and subgroup to directly examine the strength of evidence for the difference between treatment group (intervention vs control) varying between subgroups. District, cluster-level age (\% women aged 16-44 years), ethnicity (\% non-white) and socioeconomic deprivation will be the only prior defined subgroups to be considered for interaction test. Subgroup analysis will be performed regardless of the statistical significance on the overall intervention effect. The regression coefficients for the interactions between treatment group and each subgroup will be presented with the associated CIs and $p$ values.

\section{Economic evaluation}

The base case within-trial cost-effectiveness analysis will compare the NOSH offer made to women over a 6-month period postbirth versus no offer made, from a healthcare provider perspective. It will be tied to the primary outcome at $6-8$ weeks and reported as cost per percentage point change in BF rates at cluster level.

Cost will account for changes in resource use from the intervention and consequences of changes in health service use. Data collection will include (1) costs that do not vary by cluster or participant (eg, time spent setting up and negotiating coverage of the voucher scheme) and need to be apportioned to clusters; (2) costs that may vary by cluster but not by individual participant (eg, induction and training of staff); (3) costs that vary by participant (eg, number of vouchers sent, contacts made to NOSH office) that can be grouped by cluster. These data will be sourced using diaries, interviews, administrative records and the contact logging system at the NOSH office. Resource use consequences of the offer will reflect the difference in resource use impacts from hospital admissions for a range of acute conditions (GI infections, otitis media, respiratory tract infections, necrotising enterocolitis) in babies aged 0-6 months. Cluster-level HES on inpatient and emergency admissions will be converted into the relevant health resource group code using a reference costs code to group and a unit cost assigned according to the national reference costs. ${ }^{16}$ Other resource use will be valued using unit costs based on NHS reference costs ${ }^{16}$ and other national averages, for example, PSSRU 2014, ${ }^{17}$ to generate nationally generalisable estimates.

The incremental cost-effectiveness analysis will be based on regression models fitted separately for costs and the primary outcome, accounting for correlation between costs and effects and missing data where appropriate. The unit of analysis, as that of the effectiveness analysis, will be cluster level. The regression-based analyses controlling for covariates and cluster effect will be used to estimate changes in $\mathrm{BF}$, health service use and costs between trial arms.

Deterministic sensitivity and scenario analysis will explore: the impact of using all admissions rather than admissions for the four selected conditions; the potential roll-out of the NOSH scheme; and a subgroup analysis may be included if appropriate. Probabilistic sensitivity analysis will estimate precision of the costeffectiveness estimates and present cost-effectiveness acceptability curves and compute incremental net benefit statistics for specific values of decision-makers willingness to pay (WTP) for per cent-point change in $\mathrm{BF}$ rates. Beyond-trial modelling of longer term expectations for cost-effectiveness will be undertaken, with methods reported elsewhere. We will explore the possibility of generating a cost per QALY in the decision-analytic model-based analysis.

\section{Intervention process and context evaluation}

Process and context evaluation helps researchers to distinguish between results that are due to the intervention succeeding or failing, and those that may be influenced 
by the social/organisational context or implementation of the intervention. Such evaluations are especially valuable in community-based trials. ${ }^{18}{ }^{19}$ Where a cluster design is used, an additional value resides in the ability of an evaluation to assess the impact of the local context on the implementation of the intervention in each setting (cluster ward). This is likely to be particularly relevant to trials of public health initiatives as the negative consequences of the environment, resource shortages, organisational change, competing demands and leadership can affect an organisation's ability to effectively deliver an intervention. ${ }^{20} 21$ The process/context evaluation will also be used to explore any unintended consequences of the intervention.

Monitoring of the process and delivery of the intervention will be conducted using a mixture of qualitative and quantitative methods. Individual-level data will be sought on the views of healthcare providers, commissioners, funders and policymakers regarding the process of delivering the intervention and the completeness and accuracy of the routinely collected 6-8-week BF data using interviews and focus groups. Topic guide refinement, sampling strategies, and data collection and analysis will be iterative to address the specific research questions. Awareness of the intervention and views and experiences of the intervention will be collected using interviews and focus groups with mothers and social media.

\section{Qualitative data analysis}

NVivo software package will be used to enable complex organisation and retrieval of qualitative data. Framework analysis $^{22}$ will be used to analyse the data in order to enhance understanding of social phenomena in order to influence social policy in the UK. Concepts, categories and themes will be identified and coded before comparison with other data to provide analytical categories.

\section{Dissemination}

Local authority Research Governance permissions have been obtained for healthcare providers in the five trial districts to participate.

\section{TRIAL STATUS}

Data collection is ongoing.

\section{DISCUSSION}

The results of this large cRCT will be used to inform commissioners and other public health decision-makers about the acceptability, effectiveness and cost-effectiveness of behaviour change support in the form of financial incentives to mothers to breast feed in areas with low BF rates. This trial will add to the growing body of knowledge on the role of financial incentives in public health. If the intervention is found to be effective, then this would contribute to future policy discussions on how financial for $\mathrm{BF}$ might be used to improve the long-term health of the population, reduce the risk of disease and obesity in infancy, childhood and adulthood.

Author affiliations

${ }^{1}$ Public Health Section, School of Health and Related Research, University of Sheffield, Sheffield, UK

${ }^{2}$ School of Health and Related Research, University of Sheffield, Sheffield, UK ${ }^{3}$ Mother and Infant Research Unit, School of Nursing and Health Sciences, University of Dundee, Dundee, UK

${ }^{4}$ Health Economics Research Group, Brunel University London, Uxbridge, UK

Twitter Follow Mark Strong at @StrongScHARR

Acknowledgements The authors would like to thank all the mothers and healthcare providers who have contributed to the design and implementation of this study, and the National Institute for Health Research Comprehensive Research Network.

Contributors CR conceived the intervention and CR, MS, JB, MJR, SW, KT and JF-R designed the initial study. HMW, EIS, NA and SS helped with the later stages of the study design. CR wrote all drafts of the protocol with significant contributions from all authors at all stages. All authors contributed, read and approved the final manuscript.

Funding This research was funded by the Medical Research Council (MR/ J000434/1) via the National Prevention Research Initiative Phase 4 Awards. Funding for the costs of the intervention (shopping vouchers) for the trial is supported by NHS Public Health England (PHE). The NOSH Trial Steering Committee provides overall supervision of the trial and monitors trial progress and conducts and advises on scientific credibility. The Trial Steering Committee (TSC) carries the responsibility for deciding whether the trial needs to be stopped. The members are listed as follows: Dr Andrew Furber, Director of Public Health, Wakefield (Chair); Professor Andrew Briggs, Professor of Health Economics and Health Technology Assessment, Glasgow University; Professor David Tappin, Professor of Clinical Trials for Children, School of Medicine, University of Glasgow; CR, Principle Investigator (NOSH), Senior Research Fellow, ScHARR, University of Sheffield; MJR (Co-investigator), Professor of Mother and Infant Health, University of Dundee; Gavin Malloch, MRC Observer; Professor Jon Nicholl, Representative of host institution, Dean of ScHARR, Professor of Health Services Research, University of Sheffield. Director NIHR School for Public Health Research.

Disclaimer The views expressed are those of the authors and not necessarily those of the NHS or the MRC.

Competing interests None declared.

Ethics approval The study protocol has been approved by NHS and local authority Research Governance and Research Ethics Committees (NHS REC reference: 13/WM/0299)

Provenance and peer review Not commissioned; internally peer reviewed.

Open Access This is an Open Access article distributed in accordance with the Creative Commons Attribution Non Commercial (CC BY-NC 4.0) license, which permits others to distribute, remix, adapt, build upon this work noncommercially, and license their derivative works on different terms, provided the original work is properly cited and the use is non-commercial. See: http:// creativecommons.org/licenses/by-nc/4.0/

\section{REFERENCES}

1. WHO. Global strategy for infant and young child feeding. Geneva, Switzerland: World Health Organization, 2003.

2. Department of Health SSaPS. Breastfeeding and introducing solid foods: consumer insight summary. London, UK: Department of Health, 2010:31.

3. Horta BL, Bahl R, Martines JC, et al. Evidence on the long-term effects of breastfeeding: systematic review and meta-analyses. Geneva, Switzerland: Wrold Health Organisation, 2007.

4. McAndrew F, Thompson J, Fellows L, et al. Infant feeding survey 2010: summary. Health and Social Care Information Centre, 2012.

5. Kane RL, Johnson PE, Town RJ, et al. A structured review of the effect of economic incentives on consumers' preventive behavior. Am J Prev Med 2004;27:327-52. 
6. Purnell JQ, Gernes R, Stein R, et al. A systematic review of financia incentives for dietary behavior change. J Acad Nutr Diet 2014;114:1023-35

7. Groleau D, Sigouin C, D'souza NA. Power to negotiate spatial barriers to breastfeeding in a western context: when motherhood meets poverty. Health Place 2013;24:250-9.

8. Pokhrel S, Quigley M, Fox-Rushby J, et al. Potential economic impacts from improving breastfeeding rates in the UK. Arch Dis Child 2015;100:334-40.

9. Renfrew MJ, Pokhrel S, Quigley M, et al. Preventing disease and saving resources: the potential contribution of increasing breastfeeding rates in the UK. UNICEF, 2012.

10. Health Do. Improving outcomes and supporting transparency. Part 2: summary technical specifications of public health indicators. Public Health Outcomes Framework. London, UK: Department of Health, 2014:136.

11. NICE. Maternal and child nutrition. NICE public health guidance. London: National Institute for Healthcare \& Excellence, 2008:105

12. Whelan $\mathrm{B}$, Thomas $\mathrm{K}$, Van Cleemput $\mathrm{P}$, et al. Healthcare providers' views on the acceptability of financial incentives for breastfeeding: a qualitative study. BMC Pregnancy Childbirth 2014;14:355.

13. Whitford $H$, Whelan $B$, van Cleemput $P$, et al. Encouraging breastfeeding: financial incentives. Pract Midwife 2015;18:18-21.

14. Relton $\mathrm{C}$, Whelan $\mathrm{B}$, Strong $\mathrm{M}$, et al. Are financial incentives for breastfeeding feasible in the UK. A mixed methods field study. Presentation at the Lancet UK Public Health Science
Conference; November 2014, Glasgow. 2014. http://www. thelancet.com/journals/lancet/article/PIIS0140-6736\%2814\% 2962131-0/abstract

15. Campbell MK, Piaggio G, Elbourne DR, et al. Consort 2010 statement: extension to cluster randomised controlled trials. $B M J$ 2012;345:e5661.

16. Department of Health. NHS Reference Costs (2012/13). 2012. https://www.gov.uk/government/publications/nhs-referencecosts-2012-to-2013

17. Curtis L. Unit costs of health and social care. Kent: Personal Socia Services Research Unit, University of Kent, Canterbury, 2012. http:// www.pssru.ac.uk/archive/pdf/uc/uc2012/full-with-covers.pdf

18. Stapleton H, Kirkham M, Thomas G. Qualitative study of evidence based leaflets in maternity care. BMJ 2002;324:639.

19. Oakley A, Strange V, Bonell C, et al. Health services research: process evaluation in randomised controlled trials of complex interventions. BMJ 2006;332:413.

20. Hawe P, Shiell A, Riley T, et al. Methods for exploring implementation variation and local context within a cluster randomised community intervention trial. J Epidemiol Community Health 2004;58:788-93.

21. Hoddinott P, Britten J, Pill R. Why do interventions work in some places and not others: a breastfeeding support group trial. Soc Sci Med 2010;70:769-78.

22. Ritchie J, Lewis JE. Qualitative research practice: a guide for social science students and researchers. Sage Publications, 2003. 
Correction: Cluster randomised controlled trial of a financial incentive for mothers to improve breast feeding in areas with low breastfeeding rates: the NOSH study protocol

Relton C, Strong M, Renfrew MJ, et al. Cluster randomised controlled trial of a financial incentive for mothers to improve breast feeding in areas with low breastfeeding rates: the NOSH study protocol. BMJ Open 2016;6:e010158. The first and last names of the penultimate author of this paper are transposed. The author's name is 'Sue Easton'.

Open Access This is an Open Access article distributed in accordance with the Creative Commons Attribution Non Commercial (CC BY-NC 4.0) license, which permits others to distribute, remix, adapt, build upon this work noncommercially, and license their derivative works on different terms, provided the original work is properly cited and the use is non-commercial. See: http://creativecommons.org/licenses/by-nc/4.0/

BMJ Open 2016;6:e010158corr1. doi:10.1136/bmjopen-2015-010158corr1 